\title{
TURBULENT FLOWS IN CHANNELS AND PIPES
}

\author{
Khanh Chau Le \\ Lehrstuhl für Allgemeine Mechanik, \\ Ruhr-Universität Bochum, 44780 Bochum, Germany
}

\begin{abstract}
A variational principle for channel and pipe flows of incompressible viscous fluid is proposed. For low Reynolds numbers this variational principle reduces to the principle of minimum dissipation. For high Reynolds numbers it enables one to calculate the velocity profiles and the corresponding friction factors with reasonably good accuracy.
\end{abstract}

\section{INTRODUCTION}

One of long-standing issues in fluid dynamics is how to propose a unified theory of channel and pipe flows which can predict the transition from laminar to turbulent regime and simultaneously calculate the velocity profile and the friction factors for all Reynolds numbers [1]. Up to now, most of researchers in the field share the believe that this could be done by solving the Navier-Stokes equation $[2,3]$.

The present paper proposes an approach deviating largely from this main stream. It focuses on the turbulent modeling [4] rather than solving the Navier-Stokes equation. The starting point is the variational principle of minimum dissipation which is indeed the direct consequence of the Navier-Stokes equation and which can be used to determine the velocity profiles of laminar flows for small Reynolds numbers. As the Reynolds number becomes very large the flows obey asymptotically the Euler equation of the ideal fluid which again admits the variational principle $[5,6]$. The aim of this paper is to find the "bridge" between these two extreme cases. Our main assumption is that, when the Reynolds number exceeds some critical threshold, new vortices occur causing the turbulent mixing and energy and momentum transfer from large-scale to small-scale vortices. The Reynolds stress which is responsible for the momentum transfer depends on the flow generated by these emerging vortices. We attempt at formulating a variational principle involving the statistically average velocities of turbulent flow and the flow generated by new vortices by incorporating the interaction between large-scale and small-scale vortices through the energy cascade [7]. The asymptotic law of wall $[8,9]$ valid for large Reynolds numbers is used to find the nonlinear term responsible for the interaction betwcen vortices. We will show that the proposed variational principle reduces to the principle of minimum dissipation for small Reynolds number. For large Reynolds numbers it enables one to predict the velocity profile and the friction factors for turbulent flows with reasonable accuracy as compared with experimental data (see also [10]).

\section{SHEAR FLOW}

Consider an incompressible viscous fluid bounded by two parallel plates of infinite extent. The lower plate occupying the plane $y=0$ is at rest. To the upper plate occupying 
the plane $y=2 h$ the constant force $\tau$ per unit area is applied (see Fig. 1). The shear flow (or Couette flow) driven by the motion of this plate exerts the resistance to it through the viscous shear stress. If the resistance is equal to the applied force, the stationary flow will be settled. The problem is to determine the velocity of the stationary flow as function of $\tau$.

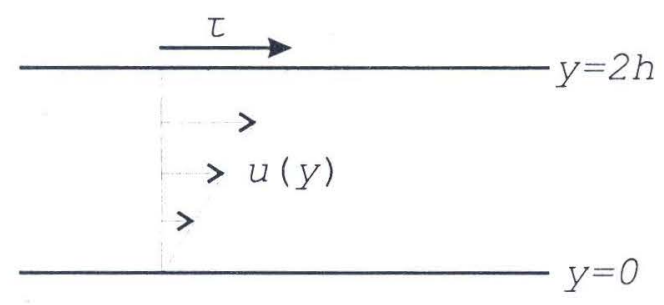

Fig. 1. Shear flow between parallel plates

It turns out that the solution of this problem exhibits extremely different behaviors at small and large $\tau$. In the laminar case (at low $\tau$ ) the solution can be found by the following variational principle: among all admissible velocity fields $u(y)$ satisfying $u(0)=0$ the true velocity field $\breve{u}(y)$ minimizes the dissipation functional

$$
\mathcal{D}=\int_{0}^{2 h} \frac{1}{2} \eta u^{\prime 2} d y-\tau u(2 h),
$$

with $\eta$ being the viscosity and the prime denoting the derivative. The first term in (2.1) describes the dissipation (per unit area), while the second term corresponds to the power of the external force and can be regarded as the energy source.

It is easy to show that the minimizer of (2.1) satisfies the equation $\eta u^{\prime}=\tau$ which leads to the linear profile of velocity $u(y)=\frac{\tau}{\eta} y$. Thus, the average velocity $\bar{u}$ is equal to the velocity in the middle of the flow at $y=h, \bar{u}=u_{m}=\tau h / \eta$. Consequently, the friction coefficient reads

$$
c_{f}=\frac{2 \tau}{\varrho u_{m}^{2}}=\frac{2}{R e},
$$

with $\varrho$ the mass density, and $R e=\varrho u_{m} h / \eta$ the Reynolds number [1]. It is more convenient to use another definition of Reynolds number which is directly related to $\tau$ (the so-called friction Reynolds number)

$$
R=\frac{\varrho u_{\tau} h}{\eta}, \quad u_{\tau}=\sqrt{\frac{\tau}{\varrho}} .
$$

Because $R e=R^{2}$ for laminar flows, we have in this case $c_{f}=2 / R^{2}$.

As the Reynolds number increases and exceeds some critical value, new vortices may occur. The energy required for the nucleation and motion of these new vortices is transferred from the energy source through the Richardson cascade [7]. Because of this energy transfer the statistically average velocity of the turbulent flow $u(y)$ is reduced considerably. For the turbulent regime many uncertainties arise except the following balance equation of mean momentum for $u(y)$ which seems to be firmly established $[4,11]$

$$
\frac{d}{d t}\left(\eta u^{\prime}+\tau F\right)=0
$$


where $\tau F$ is the so-called Reynolds stress. However, $F$ is unknown, and the problem of how to close this equation remains (see, for example, the mixing length model or the $k-\varepsilon$-model in [4]). In this paper we adopt the following two main hypotheses:

(1) Function $F$ depends only on $\varphi$ which is the statistically average velocity of the flow generated by new vortices.

(2) The governing equations for $u(y)$ and $\varphi(y)$ can be obtained from a variational principle.

Our aim is to show that the following variational principle leads to a satisfactory model for both laminar and turbulent flows: among all admissible velocity fields $u(y)$ and $\varphi(y)$ satisfying the boundary conditions

$$
u(0)=0, \quad \varphi(0)=\varphi(2 h)=0,
$$

the shear flow is described by those for which the dissipation functional

$$
\mathcal{D}=\int_{0}^{2 h}\left(\frac{1}{2} \eta u^{\prime 2}+\tau F(\varphi)\left|u^{\prime}\right|-\frac{1}{2} \eta \varphi^{\prime 2}\right) d y-\tau u(2 h)
$$

has an extremum. The first term in (2.3) is the dissipation due to viscosity, the second term describes the energy transfer from large-scale to small-scale vortices, with $F(\varphi)$ a nonlinear function of $\varphi$, the third term is responsible for the reduction in dissipation due to the energy storage by small scale vortices, and finally, the last term corresponds to the power of the external force. Here $\left|u^{\prime}\right|$ and $\left|\varphi^{\prime}\right|$ are the scalar densities of the corresponding vortices. We also assume that the wall is ideally smooth so that new vortices cannot be nucleated there. This assumption is reflected in the second boundary conditions (2.2).

It is convenient to introduce the following dimensionless quantities

$$
\zeta=\frac{R y}{h}, \quad \tilde{u}=\frac{u}{u_{\tau}}, \quad \tilde{\varphi}=\frac{\varphi}{u_{\tau}}, \quad \tilde{F}(\tilde{\varphi})=F\left(\tilde{\varphi} u_{\tau}\right), \quad \tilde{\mathcal{D}}=\frac{\mathcal{D}}{\tau u_{\tau}},
$$

with $R$ and $u_{\tau}$ being previously defined. We shall deal further only with these dimensionless quantities, therefore the tildes can be dropped for short. In this problem the velocity is monotone increasing, therefore $\left|u^{\prime}\right|=u^{\prime}$. Thus, the dimensionless dissipation becomes

$$
\mathcal{D}=\int_{0}^{2 R}\left(\frac{1}{2} u^{\prime 2}+F(\varphi) u^{\prime}-\frac{1}{2} \varphi^{\prime 2}\right) d \zeta-u(2 R) .
$$

Varying functional (2.4) we derive from it the Euler equations

$$
u^{\prime}+F(\varphi)=1, \quad F^{\prime}(\varphi) u^{\prime}+\varphi^{\prime \prime}=0 .
$$

The first equation follows directly from the balance of mean momentum and the boundary condition at $\zeta=2 R$. Expressing $u^{\prime}$ through $F(\varphi)$ in the first equation and substituting it into the second equation we obtain the governing equation for $\varphi$

$$
\varphi^{\prime \prime}+F^{\prime}(\varphi)(1-F(\varphi))=0
$$

Equation (2.6) has the form of the equation of motion of a particle with mass 1 in the potential well

$$
V(\varphi)=F(\varphi)-\frac{1}{2} F^{2}(\varphi) .
$$

The immediate consequence of this is the first integral

$$
\frac{1}{2} \varphi^{\prime 2}+V(\varphi)=V\left(\varphi_{m}\right)
$$


where $\varphi_{m}$ is the maximal velocity of the vortex flow which is achieved at $\zeta=R$. We assume that both $F(\varphi)$ and $V(\varphi)$ are even function of $\varphi$.

The determination of $F(\varphi)$ is based on the law of wall $[8,9]$. This law states that for Reynolds numbers approaching infinity the Reynolds stress $F\left(\varphi_{\infty}(\xi)\right)$ becomes a universal function $f(\xi)$, with $\xi \in(0, \infty)$ the so-called "wall coordinate". From various experimental data (see $[4,11]$ we know that $f(\xi) \sim 1-\frac{1}{\kappa \xi}$ as $\xi \rightarrow \infty$, with $\kappa=0.41$ the Karman constant, and $f(\xi) \sim a \xi^{3}$ for small $\xi$. We use therefore the following semi-empirical formula

$$
f(\xi)=1-\frac{1}{1+(a+b) \xi^{3}}-\frac{b \xi^{3}}{1+\kappa b \xi^{4}},
$$

for this universal function, with $a=6.10^{-4}, b=1.08510^{-6}$ (compare with [11]). Thus,

$$
\frac{1}{2} \varphi_{\infty}^{\prime 2}+V\left(\varphi_{\infty}(\xi)\right)=\frac{1}{2} \Rightarrow \varphi_{\infty}^{\prime}(\xi)=\sqrt{1-2 V(\varphi(\xi))}=1-f(\xi) .
$$

Equations (2.5) 1 and (2.10) show that for very large Reynolds numbers the vorticity densities of turbulent and vortex flows are equal, what seems to be quite natural. With function $f(\xi)$ from $(2.9)$ we find

$$
\begin{array}{r}
\varphi_{\infty}(\xi)=\frac{1}{\Lambda}\left[\frac{1}{3} \ln \frac{\Lambda \xi+1}{\sqrt{(\Lambda \xi)^{2}-\Lambda \xi+1}}+\frac{1}{\sqrt{3}}\left(\arctan \frac{2 \Lambda \xi-1}{\sqrt{3}}+\frac{\pi}{6}\right)\right] \\
+\frac{1}{4 \kappa} \ln \left(1+\kappa b \xi^{4}\right)
\end{array}
$$

where $\Lambda=(a+b)^{1 / 3}$. The one-to-one correspondence between $\varphi$ and $\xi$ can be achieved

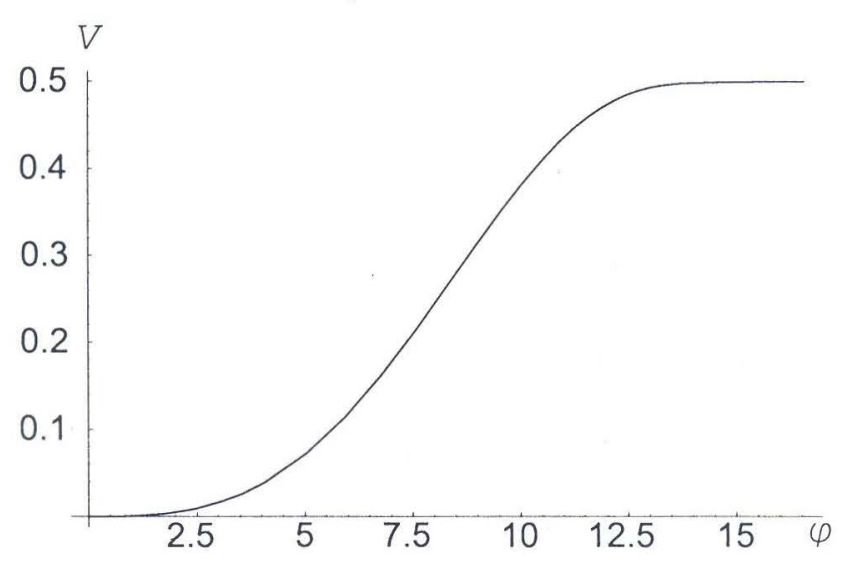

Fig. 2. Function $V(\varphi)$

by setting $\varphi_{\infty}(\xi)=-\varphi_{\infty}(-\xi)$ for $\xi<0$. This velocity profile possesses the following remarkable asymptotic property: $\varphi_{\infty}(\xi) \sim \ln \xi / \kappa+c$ as $\xi \rightarrow \infty$, where $c=5.41$. The plot of $V(\varphi)$ using $\xi$ as parameter is shown in Fig. 2. This function applies to all Reynolds numbers. It is interesting to mention that function $V(\varphi)$ behaves like $a \varphi^{3}$ for small $\varphi$ and $1-e^{-2 \kappa \varphi} / \kappa^{2}$ for large $\varphi$. 


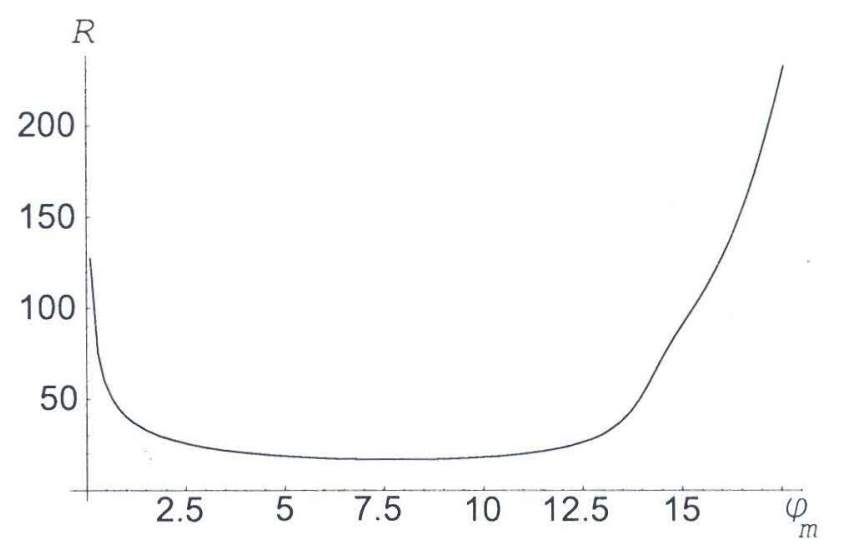

Fig. 3. Reynolds number as function of $\varphi_{m}$

Knowing $V(\varphi)$ one can integrate equation (2.8) to determine $\varphi$ and then $u$. In terms of the wall coordinates $\xi$ we have

$$
R=\int_{0}^{\xi_{m}} \frac{[1-f(\xi)] d \xi}{\sqrt{2\left[v\left(\xi_{m}\right)-v(\xi)\right]}}
$$

where $v(\xi)=f(\xi)-\frac{1}{2} f^{2}(\xi)$. It is easy to show that $R$ tends to infinity as $\xi_{m}$ tends to zero like $R \sim \sqrt{\frac{\pi}{2 a \xi_{m}}} \frac{\Gamma(4 / 3)}{\Gamma(5 / 6)}$, and tends to infinity as $\xi_{m}$ tends to infinity like a linear function. It has one minimum $R_{c}=16.9714$ which is achieved at $\xi_{m}=8.4397$ (or $\varphi_{m}=7.805$ ). For $R<R_{c}$ the extremal $\varphi$ must vanish. Thus, the value $R_{c}=16.9714$ can be regarded as the critical Reynolds number, at which the transition from laminar to turbulent shear flow takes place (this corresponds to $R e_{c}=288.028$ ). The plot of $R$ as function of $\varphi_{m}$ is shown in Fig. 3. One can see that the laminar flow is stable in "small" and that the transition from laminar to turbulent regime is "hard" in the sense that a finite amplitude of velocity of vortex motion is required for it. For example, if the disturbances of velocity of vortex motion is smaller than 0.163 , then the laminar regime can be maintained up to the Reynolds number $R=100$. This agrees qualitatively with the stability analysis of the Navier-Stokes equation [12].

Finally we find the distribution of velocity $u(\zeta)$ of turbulent flow from $\varphi(\zeta)$ by integrating the equation $(2.5)_{1}[10]$. The plot of the difference $d=u_{m}-\ln R / \kappa$, with $u_{m}$ being the velocity in the middle of the flow, as function of $R$ is shown in Fig. 4. As $R$ tends to infinity, this difference tends to the value 7.1 which coincides with the empirical value given in [11].

\section{CHANNEL FLOW}

Consider next the 2-D flow of the incompressible viscous fluid in a channel driven by the constant pressure gradient (Poiseuille flow). Both plates are now at rest. In the laminar case (low pressure gradient) the velocity $u(y)$ is the minimizer of the dissipation 


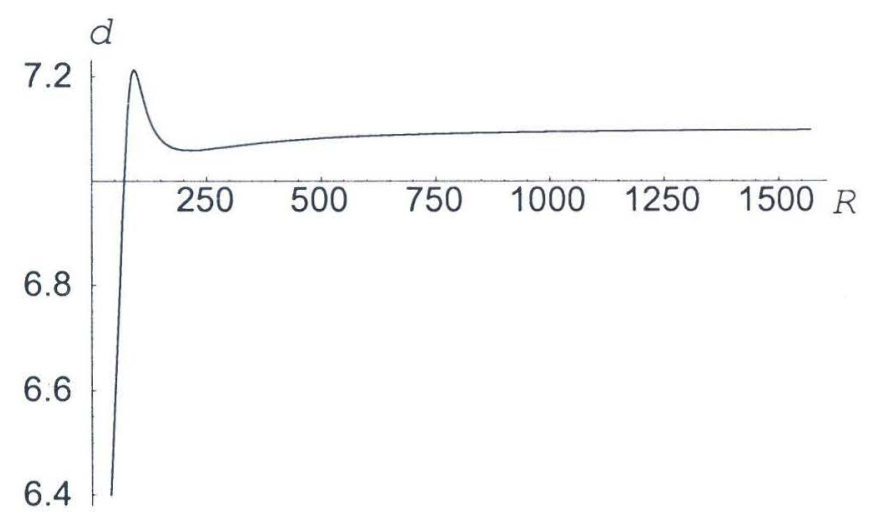

Fig. 4. Difference $d=u_{m}-\ln R / \kappa$ versus Reynolds number $R$

functional

$$
\mathcal{D}=\int_{-h}^{h} \frac{1}{2} \eta u^{\prime 2} d y+p^{\prime} \int_{-h}^{h} u(y) d y
$$

under the constraint

$$
u( \pm h)=0
$$

To make the symmetry more transparent, the origin is now chosen in the middle of the flow, and we let $p^{\prime}$ denote the pressure gradient with respect to $x$ which is constant over the crosssection. It is easy to show that the velocity profile is parabolic: $u(y)=-\frac{p^{\prime}}{2 \eta}\left(h^{2}-y^{2}\right)$, so the average velocity is equal to $\bar{u}=-p^{\prime} h^{2} / 3 \eta$. This yields the following friction coefficient

$$
c_{f}=\frac{-p^{\prime} h}{\frac{1}{2} \varrho \bar{u}^{2}}=\frac{18}{R^{2}},
$$

where $R=\varrho u_{p} h / \eta, u_{p}=\sqrt{-p^{\prime} h / \varrho}$.

As the pressure gradient becomes large, a flow induced by new vortices occurs. We let $u(y)$ denote as before the statistically average velocity of turbulent flow and $\varphi(y)$ the statistically average velocity of flow generated by new vortices. Again, there are many uncertainties in the turbulent regime except the firmly established balance equation of mean momentum $[4,11]$

$$
\frac{d}{d y}\left(\eta u^{\prime}+p^{\prime} h F\right)=p^{\prime}
$$

where $p^{\prime} h F$ corresponds to the Reynolds stress describing the momentum transfer by the turbulent mixing. Adopting the same hypotheses as in the previous case, we try to close this equation by formulating the following variational principle: among all admissible velocity fields $u(y)$ and $\varphi(y)$ satisfying the boundary conditions

$$
u( \pm h)=0, \quad \varphi( \pm h)=0,
$$

the channel flow is described by those for which the dissipation functional

$$
\mathcal{D}=\int_{-h}^{h}\left(\frac{1}{2} \eta u^{\prime 2}-p^{\prime} h F(\varphi)\left|u^{\prime}\right|+p^{\prime} h \frac{\alpha}{R^{2 / 5}}\left|u^{\prime}\right|-\frac{1}{2} \eta \varphi^{\prime 2}\right) d y+p^{\prime} \int_{-h}^{h} u(y) d y
$$


has an extremum. In comparison with the shear flow the only new term added in this dissipation functional is the third term which is responsible for the energy storage of large scale vortices, where $\alpha$ is now the universal parameter which is chosen to be equal to $\alpha=1.442$. It will be shown that the $2 / 5$ scaling law yields the satisfactory agreement with the experimental data.

By changing the variables and unknown functions

$$
\zeta=\frac{R y}{h}, \quad u \mapsto \frac{u}{u_{p}}, \quad \varphi \mapsto \frac{\varphi}{u_{p}}, \quad \mathcal{D} \mapsto-\frac{\mathcal{D}}{p^{\prime} h u_{p}},
$$

we transform the dissipation functional to

$$
\mathcal{D}=\int_{-R}^{R}\left(\frac{1}{2} u^{\prime 2}+F(\varphi)\left|u^{\prime}\right|-\frac{\alpha}{R^{2 / 5}}\left|u^{\prime}\right|-\frac{1}{2} \varphi^{\prime 2}\right) d \zeta-\int_{-R}^{R} \frac{1}{R} u(\zeta) d \zeta .
$$

Note that the extremal possesses the following symmetry property: $u(\zeta)$ is even and $\varphi(\zeta)$ is odd function with respect to $\zeta$. Due to this symmetry we shall consider the half-interval $(-R, 0)$ where $u^{\prime}(\zeta) \geq 0$.

Observe first that the variational problem (3.3) always has the extremal $\varphi=0$ leading to the laminar velocity profile. However, for sufficiently large $R$ there is another extremal describing the turbulent flow. For the developed turbulent flow (large $R$ ) we assume that

$$
u(\zeta)= \begin{cases}u_{1}(\zeta), \quad \zeta \in(-R,-l), \\
u_{m}, \quad \zeta \in(-l, l), \quad \varphi(\zeta)=\left\{\begin{array}{ll}
\varphi_{1}(\zeta), & \zeta \in(-R,-l), \\
u_{1}(-\zeta), & \zeta \in(l, R),
\end{array} \quad, \quad \zeta \in(-l, l),\right. \\
\left.-\varphi_{1}(\zeta), \zeta\right), \quad \zeta \in(l, R),\end{cases}
$$

with $u_{m}=$ const and $l$ being the unknown length which must be subject to variation. This assumption means that the flow in the middle of the channel can be regarded as the flow of ideal fluid. Then the half-dissipation becomes

$$
\begin{aligned}
\frac{\mathcal{D}}{2}=\int_{-R}^{-l}\left[\frac{1}{2} u_{1}^{\prime 2}+F\left(\varphi_{1}\right) u_{1}^{\prime}-\frac{\alpha}{R^{2 / 5}} u_{1}^{\prime}\right. & \left.-\frac{1}{2} \varphi_{1}^{\prime 2}\right] d \zeta-\int_{-l}^{0} \frac{1}{2} \varphi_{m}^{\prime 2} d \zeta \\
& -\int_{-R}^{-l} \frac{1}{R} u_{1}(\zeta) d \zeta-\frac{u_{m} l}{R} .
\end{aligned}
$$

The standard calculus of variation leads to the differential equations

$$
\begin{aligned}
-\frac{d}{d \zeta}\left(u^{\prime}+F(\varphi)\right)-\frac{1}{R} & =0, \\
F^{\prime}(\varphi) u^{\prime}+\varphi^{\prime \prime} & =0,
\end{aligned}
$$

for $\zeta \in(-R,-l)$ and

$$
\varphi^{\prime \prime}=0
$$

for $\zeta \in(-l, 0)$. The boundary conditions read

$$
\begin{aligned}
u(-R) & =\varphi(-R)=0, \quad \varphi(0)=0, \\
u^{\prime}(-l)=0, \quad \varphi^{\prime}(-l+0)=\varphi^{\prime}(-l-0), \quad F(\varphi(-l))-\frac{\alpha}{R^{2 / 5}} & =\frac{l}{R} .
\end{aligned}
$$

Two first boundary conditions in $(3.7)_{2}$ mean the continuity of the vorticity densities. The last condition is obtained by varying $u_{m}$, where the influence of the third term in $(3.3)$ becomes noticeable for extremals with the assumed behavior. 
Equation (3.5) 1 and the last boundary condition of (3.7) imply that

$$
u^{\prime}+F(\varphi)=-\frac{\zeta}{R}+\frac{\alpha}{R^{2 / 5}} .
$$

Expressing $u^{\prime}$ through $F(\varphi)$ and substituting into the second equation of (3.5) we obtain the following governing equation for $\varphi$ in $(-R,-l)$

$$
\varphi^{\prime \prime}-F^{\prime}(\varphi)\left(\frac{\zeta}{R}-\frac{\alpha}{R^{2 / 5}}+F(\varphi)\right)=0 .
$$

This equation is subject to the boundary conditions

$$
\varphi(-R)=0, \quad F(\varphi(-l))=\frac{l}{R}+\frac{\alpha}{R^{2 / 5}}, \quad \varphi^{\prime}(-l)=-\frac{\varphi(-l)}{l} .
$$

The last boundary condition is the direct consequence of the equation (3.6), the boundary condition $\varphi(0)=0$, and the continuity of $\varphi^{\prime}$.

Since $F(\varphi)$ does not have the explicit analytical form, it is convenient to choose the wall coordinate $\xi(\zeta)$ as unknown function in accordance to

$$
\begin{array}{r}
\varphi(\zeta)=\varphi_{\infty}(\xi(\zeta)), \quad F(\varphi)=f(\xi), \\
\varphi^{\prime}=(1-f(\xi)) \xi^{\prime}, \quad \varphi^{\prime \prime}=(1-f(\xi)) \xi^{\prime \prime}-f^{\prime}(\xi) \xi^{\prime 2} .
\end{array}
$$

In terms of the wall coordinate the governing equation becomes

$$
(1-f(\xi)) \xi^{\prime \prime}-f^{\prime}(\xi) \xi^{\prime 2}-\frac{f^{\prime}(\xi)}{1-f(\xi)}\left(\frac{\zeta}{R}-\frac{\alpha}{R^{2 / 5}}+f(\xi)\right)=0 .
$$

This equation is subject to the boundary conditions

$$
\xi(-R)=0, \quad \xi(-l)=f^{-1}\left(\frac{l}{R}+\frac{\alpha}{R^{2 / 5}}\right), \quad \xi^{\prime}(-l)=-\frac{\varphi_{\infty}\left(f^{-1}\left(\frac{l}{R}+\frac{\alpha}{R^{2 / 5}}\right)\right)}{l\left(1-\frac{l}{R}-\frac{\alpha}{R^{2 / 5}}\right)},
$$

where $f^{-1}$ is the inverse function of $f$. Equation (3.12) can also be directly derived from the variational principle (3.4).

The shooting method can be applied to find the solution of the two-point boundaryvalue problem (3.12), (3.13). Knowing $u^{\prime}(\zeta)$ we can find the velocity profile $u(\zeta)$ and then the average velocity $\bar{u}[10]$. Fig. 5 presents the graph of $d=\bar{u}-\frac{\ln R}{\kappa}$ versus the Reynolds number $R$. For $R=1000$ the theory predicts $d=3.09$ which is close to the empirical value 3.3 taken from [11].

\section{PIPE FLOW}

For the laminar pipe flow driven by the constant pressure gradient (3-D Poiseuille flow) the velocity $u(r)$ is the minimizer of the dissipation functional

$$
\mathcal{D}=\int_{0}^{a} \frac{1}{2} \eta u^{\prime 2} 2 \pi r d r+p^{\prime} \int_{0}^{a} u(r) 2 \pi r d r
$$

under the constraint

$$
u(a)=0 .
$$

Here the polar coordinate $r$ is used, with $a$ the radius of the circular cross-section, and $p^{\prime}$ is the pressure gradient which is constant over the cross-section. It is easy to show that the 


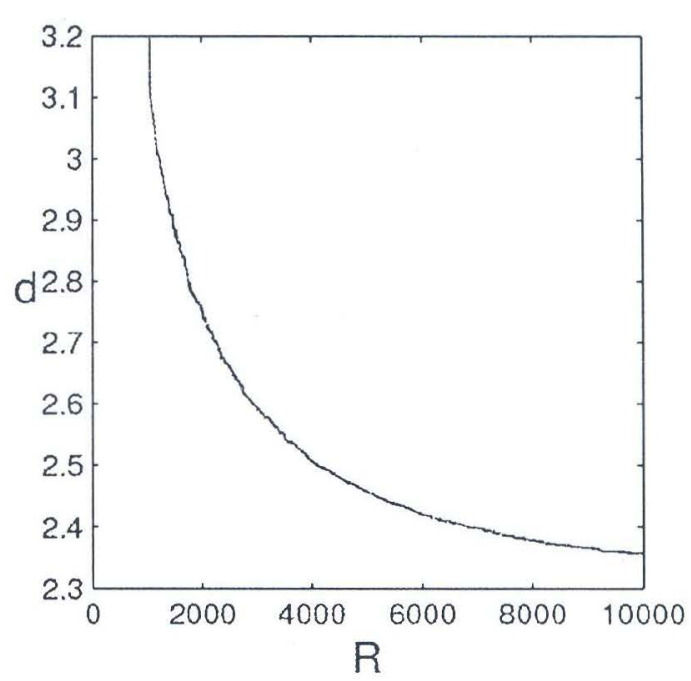

Fig. 5. Difference $d=\bar{u}-\ln R / \kappa$ versus Reynolds number $R$

velocity profile is parabolic: $u(r)=-\frac{p^{\prime}}{4 \eta}\left(a^{2}-r^{2}\right)$, which leads to the following resistance law

$$
c_{f}=\frac{-p^{\prime} a}{\frac{1}{2} \varrho \bar{u}^{2}}=\frac{64}{R^{2}}
$$

where $R=\varrho u_{p} a / \eta, u_{p}=\sqrt{-p^{\prime} a / 2 \varrho}$.

As the pressure gradient becomes large, a flow induced by new vortices occurs. We let $u(r)$ denote as before the statistically average velocity of turbulent flow and $\varphi(r)$ the statistically average velocity of flow generated by new vortices. Similar to the previous cases, the only firmly established equation is the balance of mean momentum

$$
\frac{1}{r} \frac{d}{d r}\left(r\left(\eta u^{\prime}+\frac{1}{2} p^{\prime} a F\right)\right)=p^{\prime}
$$

where $\frac{1}{2} p^{\prime} a F$ corresponds to the Reynolds stress. Since $F$ is unknown, one needs to close this equation. Adopting the same hypotheses as before, we formulate the following variational principle: among all admissible velocity fields $u(r)$ and $\varphi(r)$ satisfying the boundary conditions

$$
u(a)=0, \quad \varphi(a)=0,
$$

the pipe flow is described by those for which the dissipation functional

$$
\mathcal{D}=\int_{0}^{a}\left(\frac{1}{2} \eta u^{\prime 2}-\frac{p^{\prime} a}{2} F(\varphi)\left|u^{\prime}\right|+\frac{p^{\prime} a}{2} \frac{\alpha}{R^{2 / 5}}\left|u^{\prime}\right|-\frac{1}{2} \eta \varphi^{\prime 2}\right) 2 \pi r d r+p^{\prime} \int_{0}^{a} u(r) 2 \pi r d r
$$

has an extremum, where, as before, $\alpha=1.442$.

By changing the variables and unknown functions

$$
\zeta=\frac{R r}{a}, \quad u \mapsto \frac{u}{u_{p}}, \quad \varphi \mapsto \frac{\varphi}{u_{p}}, \quad \mathcal{D} \mapsto-\frac{\mathcal{D} R}{\pi p^{\prime} a^{2} u_{p}}
$$


we transform the dissipation functional to

$$
\mathcal{D}=\int_{0}^{R}\left(\frac{1}{2} u^{\prime 2}+F(\varphi)\left|u^{\prime}\right|-\frac{\alpha}{R^{2 / 5}}\left|u^{\prime}\right|-\frac{1}{2} \varphi^{\prime 2}\right) \zeta d \zeta-\int_{0}^{R} \frac{2}{R} u(\zeta) \zeta d \zeta .
$$

The variational problem (4.3) always has the extremal $\varphi=0$ leading to the laminar velocity profile. However, for sufficiently large $R$ there is another extremal describing the turbulent flow. For the developed turbulent flow (large $R$ ) we assume that

$$
u(\zeta)=\left\{\begin{array}{ll}
u_{m} & \text { for } \zeta \in(0, l), \\
u_{1}(\zeta) & \text { for } \zeta \in(l, R),
\end{array}, \quad \varphi(\zeta)= \begin{cases}\varphi_{m}(\zeta) & \text { for } \zeta \in(0, l), \\
\varphi_{1}(\zeta) & \text { for } \zeta \in(l, R),\end{cases}\right.
$$

with $u_{m}=$ const and $l$ being the unknown length which must be subject to variation. Thus, the flow in the middle of the pipe can be regarded as the flow of ideal fluid. Then

$$
\begin{array}{r}
\mathcal{D}=-\int_{0}^{l} \frac{1}{2} \varphi_{m}^{\prime 2} \zeta d \zeta+\int_{l}^{R}\left[\frac{1}{2} u_{1}^{\prime 2}-F\left(\varphi_{1}\right) u_{1}^{\prime}+\frac{\alpha}{R^{2 / 5}} u_{1}^{\prime}-\frac{1}{2} \varphi_{1}^{\prime 2}\right] \zeta d \zeta \\
-\frac{u_{m} l^{2}}{R}-\int_{l}^{R} \frac{2}{R} u_{1}(\zeta) \zeta d \zeta .
\end{array}
$$

By the similar method we derive the differential equations

$$
\left(\varphi^{\prime} \zeta\right)^{\prime}=0
$$

for $\zeta \in(0, l)$ and

$$
\begin{aligned}
-\frac{d}{d \zeta}\left[\left(u^{\prime}-F(\varphi)+\frac{\alpha}{R^{2 / 5}}\right) \zeta\right]-\frac{2 \zeta}{R} & =0, \\
-F^{\prime}(\varphi) u^{\prime} \zeta+\left(\varphi^{\prime} \zeta\right)^{\prime} & =0,
\end{aligned}
$$

for $\zeta \in(l, R)$. The boundary conditions read

$$
\begin{array}{r}
\varphi(0)=0, \quad u(R)=\varphi(R)=0, \\
u^{\prime}(l)=0, \quad \varphi^{\prime}(l+0)=\varphi^{\prime}(l-0), \quad F(\varphi(l))-\frac{\alpha}{R^{2 / 5}}=\frac{l}{R} .
\end{array}
$$

The last condition is obtained by varying $u_{m}$.

Equation (4.6) 1 and the last boundary condition of (4.7) imply that

$$
u^{\prime}-F(\varphi)+\frac{\alpha}{R^{2 / 5}}=-\frac{\zeta}{R} .
$$

Expressing $u^{\prime}$ through $F(\varphi)$ and substituting into the second equation of (4.6) we obtain the following governing equation for $\varphi$ in $(l, R)$

$$
\left(\varphi^{\prime} \zeta\right)^{\prime}-F^{\prime}(\varphi)\left(F(\varphi)-\frac{\zeta}{R}-\frac{\alpha}{R^{2 / 5}}\right) \zeta=0
$$

This equation is subject to the boundary conditions

$$
F(\varphi(l))=\frac{l}{R}+\frac{\alpha}{R^{2 / 5}}, \quad \varphi^{\prime}(l)=0, \quad \varphi(R)=0 .
$$

The second boundary condition is the direct consequence of the equation (4.5), the boundary condition $\varphi(0)=0$, and the continuity of $\varphi^{\prime}$. 
In terms of the wall coordinate the governing equation becomes

$$
(1-f(\xi)) \xi^{\prime \prime} \zeta-f^{\prime}(\xi) \xi^{2} \zeta+(1-f(\xi)) \xi^{\prime}-\frac{f^{\prime}(\xi)}{1-f(\xi)}\left(f(\xi)-\frac{\zeta}{R}-\frac{\alpha}{R^{2 / 5}}\right) \zeta=0 .
$$

This equation is subject to the boundary conditions

$$
\xi(l)=f^{-1}\left(\frac{l}{R}+\frac{\alpha}{R^{2 / 5}}\right), \quad \xi^{\prime}(l)=0, \quad \xi(R)=0 .
$$

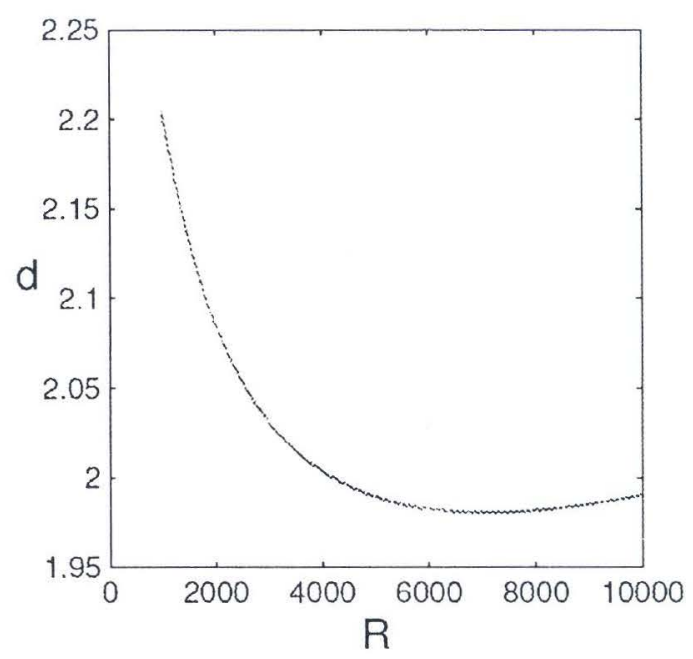

Fig. 6. Difference $d=\bar{u}-\ln R / \kappa$ versus Reynolds number $R$

By using the shooting method one can find the velocity profile $u(\zeta)$ and then the average velocity $\bar{u}[10]$. In Fig. 6 the plot of $d=\bar{u}-\ln R / \kappa$ versus the Reynolds number $R$ is shown. For the Reynolds number $R=10000$ the numerical calculations yield $\bar{u}=$ 24.4576 and $d=1.9934$. This is in excellent agreement with the empirical value 1.96. Introducing the skin-friction coefficient $c_{f}$, the friction factor $\lambda$ and the Reynolds number Re in accordance to

$$
c_{f}=\frac{\lambda}{4}=\frac{-2 p^{\prime} a}{\varrho \bar{u}^{2}}, \quad R e=\frac{2 \varrho \bar{u} a}{\eta}
$$

we derive from here the well-known Prandtl's friction law for turbulent flows

$$
\frac{1}{\lambda}=2 \log _{10}(\operatorname{Re} \sqrt{\lambda})-0.8 \text {. }
$$

\section{CONCLUSIONS}

The unified theory of channel and pipe flows proposed in this paper is based on the following hypotheses:

- The Reynolds stress depends on the mean velocity of the flow generated by new vortices

- The governing equations for the mean velocities of the turbulent flow and of the flow generated by new vortices can be obtained from a variational principle

- The asymptotic law of wall holds true for large Reynolds numbers. 
It is shown that this theory leads to the satisfactory description of channel and pipe flows for both laminar and turbulent regimes. For large Reynolds numbers the theory predicts the velocity profile and the friction factors with reasonable accuracy as compared with experimental data.

\section{REFERENCES}

1. O. Reynolds. An experimental investigation of the circumstances which determines whether the motion of water shall be direct or sinuous, and of the law of resistance in parallel channels, Phil. Trans. Roy. Soc. London 174 (1983) 935-982.

2. M. Navier. Memoire sur les lois du mouvement des fluides. Mem. de l'Acad. d. Sci. 6 (1827) 389-416.

3. G. G. Stokes. On the theory of the internal friction of fluids in motion, and of the equilibrium and motion of elastic solids. Trans. Cambr. Phil. Soc. 8 (1949) 287-319.

4. S. B. Pope. Turbulent Flows, Cambridge University Press, Cambridge, 2000.

5. V. I. Arnold. Variational principle for three-dimensional stationary flows of ideal fluid, Appl. Math. Mech. (PMM) 29 (1965) 846-851.

6. V. L. Berdichevsky. Variational principles of continuum mechanics. Nauka, Moscow, 1983.

7. A. H. Kolmogorov. The local structure of turbulence in incompressible viscous fluid for very large reynolds number. Dokl. Akad. Nauk SSSR 30 (1941) 299-303.

8. T. von Karman. Mechanische Änhlichkeit und Turbulenz. Nachr. Ges. Wiss. Göttingen, Math.Phys. Kl., pages 58-76, 1930.

9. L. Prandtl. Zur turbulenten Strömung in Röhren und längs Platten, Ergebn. Aerodyn. Versuchsanst. 4 (1932) 18-29.

10. K. C. Le. A unified theory of channel and pipe flows, WSEAS Trans. Fluid Mech., 1 (2006) $785-792$.

11. H. Schlichting and K. Gersten, Grenzschicht-Theorie, Springer Verlag, Berlin, 1997.

12. C. C. Lin, The Theory of Hydrodynamic Stability, Cambridge University Press, Cambridge, 1955.

Received June 5, 2007.

\section{DÒNG RỐI TRONG KÊNH VÀ ỐNG}

Nguyên lý biến phân đối vói các dòng chất lơng nhớt không nén được trong kênh và ống được đề xuất. Đối với những số Reynolds thấp nguyên lý biến phân rút gọn về nguyên lý hao tán cực tiểu. Đối với các số Reynolds cao có thể tính profile vận tốc và hệ số ma sát tương ứng với độ chính xác khá hợp lý. 